\title{
New strategies to improve exercise tolerance in chronic obstructive pulmonary disease
}

\author{
N. Ambrosino, S. Strambi
}

New strategies to improve exercise tolerance in chronic obstructive pulmonary disease. N. Ambrosino, S. Strambi. C ERS Journals Ltd 2004.

ABSTRACT: Breathlessness is the most common symptom limiting exercise in patients with chronic obstructive pulmonary disease (COPD). Exercise training can improve both exercise tolerance and health status in these patients, intensity being of key importance. Nevertheless, in these patients extreme breathlessness and/or peripheral muscle fatigue may prevent patients from higher levels of intensity. In this review article the literature concerning the different ways to optimise exercise tolerance in patients with COPD, with the objective of enhancing the tolerance to higher exercise training intensity, is summarised.

Continuous positive airway pressure and different modalities of noninvasive positive pressure ventilation (NPPV) may reduce breathlessness and increase exercise tolerance in these patients. Respiratory muscle unloading and reduction in intrinsic positive endexpiratory pressure have been considered among mechanisms underlying these effects. Nevertheless, the role of NPPV in pulmonary rehabilitation, if any, is still controversial. The addition of nocturnal domiciliary NPPV during a daily exercise programme in patients with severe COPD resulted in an improvement in exercise tolerance and quality of life.

In patients with severe COPD application of electrical stimulation combined with active limb mobilisation significantly improved muscle strength, and interval training has been shown capable of inducing physiological training effects.

Oxygen supplementation in patients who do not desaturate during exercise seems to be the most promising treatment, since it allows for higher exercise intensities and, therefore, superior training efficacy.

In conclusion, further studies are needed to define the appropriate patients in order to generalise such interventions. The modalities discussed should be used as adjuncts to a well designed comprehensive respiratory rehabilitation programme.

Eur Respir J 2004; 24: 313-322.
Pulmonary Unit, Cardio-Thoracic Dept, University Hospital, Pisa, Italy.

Correspondence: N. Ambrosino

Pulmonary Unit

Cardio-Thoracic Dept

Azienda Ospedaliera

Universitaria Pisana

Via Paradisa 2

Cisanello

57124 Pisa

Italy

Fax: 39050996779

E-mail: n.ambrosino@ao-pisa.toscana.it

Keywords: Electrical stimulation

interval training

mechanical ventilation

oxygen

pulmonary rehabilitation

respiratory muscles

Received: January 92004

Accepted after revision: March 82004
Only long-term oxygen therapy (LTOT) and smoking cessation improve survival in patients with chronic obstructive pulmonary disease (COPD) [1, 2]. COPD is a heterogeneous disorder characterised by dysfunction of the small and large airways, as well as by destruction of the lung parenchyma and vasculature, in highly variable combinations [3]. Breathlessness and exercise intolerance are the most common symptoms in COPD and progress relentlessly as the disease advances. In these patients, although the primary pathological changes are confined to the lungs, the consequent physical deconditioning and emotional responses contribute a large extent to morbidity. Increased breathlessness leads to inactivity and consequent peripheral muscle deconditioning, resulting in a vicious cycle leading to further inactivity, social isolation, fear of dyspnoea and depression. Patients with severe COPD become less mobile and reduce their activities of daily living (ADL). In a survey of patients with severe COPD treated with LTOT, 50\% (Medical Research Council dyspnoea grade 5) did not leave the house and $78 \%$ were breathless walking around at home and performing ADL [4]. Multidisciplinary pulmonary rehabilitation may offer a useful tool [5]. In fact it has been shown that the utilisation of healthcare services by COPD patients is related more to respiratory and peripheral muscle force than to airway obstruction [6]. Moreover, in patients hospitalised with an acute exacerbation of severe COPD, 1-yr survival was reported to be independently related to nutritional status, as assessed by body mass index, and prior functional status [7]. Multidisciplinary rehabilitation can positively influence peripheral and respiratory muscle function, nutrition and ADL [8-11].

\section{Mechanism of exercise dyspnoea}

Pathophysiological factors known to contribute to exertional dyspnoea in COPD patients include increased intrinsic mechanical loading of inspiratory muscles, i.e. the intrinsic positive end-expiratory pressure (PEEPi), (the inspiratory threshold load (ITL)) [12], increased mechanical restriction of the thorax, inspiratory muscle weakness, increased ventilatory demand relative to capacity, gas exchanges abnormalities, dynamic airway compression, cardiovascular factors, and any combination of the above [13]. Besides lung function, peripheral muscle force is also an important determinant of exercise capacity in COPD. Peripheral muscle wasting is a 
common finding in advanced COPD, as in several other chronic diseases [14]. Recent advances in clinical research have confirmed the negative impact of muscle wasting on patients' survival [15], and, at the same time, improved understanding how muscle mass is maintained [16, 17]. The consequences of peripheral muscle wasting on therapeutic approaches should be taken into account. Gain in muscle mass and strength has been associated with better exercise tolerance and survival [15], therefore, improving peripheral muscle function could be a reasonable therapeutic target in patients with COPD. Pharmacological approach to this problem (e.g. anabolic steroid [18] and growth hormone [19] supplementation) is still questioned. Most probably, exercise training is the best available therapeutic modality to preserve muscle mass, since physical activity can decrease proteasome activity [20] and muscle redox potential in COPD [21].

\section{Exercise training}

Patients with COPD can achieve a physiological training effect from well-designed programmes of exercise training as a part of comprehensive pulmonary rehabilitation programmes. Patients with mild COPD respond to a programme of high intensity training with reduced levels of blood lactate and pulmonary ventilation at a given heavy work rate [22]. Substantial improvements in exercise tolerance can be obtained as a result of programmes of exercise training even in those patients with severe obstruction who are unable to elevate blood lactate levels. The improvements of exercise tolerance have been found to be accompanied by physiological changes, such as improved muscle function (including more rapid oxygen uptake kinetics following exercise onset) [23] and altered breathing pattern (higher tidal volume $(V \mathrm{~T})$ and lower breathing frequency that lead to a reduced dead space to $V \mathrm{~T}$ ratio and thus to a lower ventilatory requirement for exercise [24]). Intensive training increases the levels of aerobic enzymes and the capillary density of leg muscle of patients with severe COPD as assessed by morphological and biochemical analysis of muscle biopsy specimens of thigh muscle $[25,26]$.

\section{Aim of review}

Given the demonstrated value of exercise training [27, 28], recent research has focused on methods to enhance the effectiveness of exercise training and, in addition, to define new approaches to improving muscle function. The rationale for such approaches is based on the above mechanisms of reduced exercise tolerance of COPD, namely respiratory muscle overburden and reduced peripheral muscle function. In this review article the literature concerning the different ways to optimise exercise tolerance in patients with COPD is summarised with the objective of enhancing the tolerance to higher exercise training intensity. Reviewing literature from 1980-2004 (PubMed) shows 986 articles under the terms COPD and rehabilitation. For the purpose of this review articles have been reviewed according the following terms: COPD and exercise and: 1) mechanical ventilation: 61 studies, including one methanalisis; 2) electrical stimulation: five studies; 3) interval training: 16 articles including two methanalisis; 4) oxygen: 101 studies; and 5) biofeedback: four studies.

\section{Noninvasive ventilation and exercise}

Exercise training as a part of multidisciplinary pulmonary rehabilitation can improve both exercise tolerance and healthrelated quality of life (HRQL) in COPD patients [27, 28].
Physiological changes contribute to these improvements: reduction of lactic acidosis, minute ventilation and heart rate for a given work rate, and enhanced activity of mitochondrial enzymes and capillary density in the trained muscles. Intensity of exercise training is of key importance. High-intensity training improved both maximal and submaximal exercise tests and induced both cardio-respiratory and peripheral muscle adaptations [22, 29, 30]. Nevertheless, in these patients extreme breathlessness and/or peripheral muscle fatigue may prevent patients from higher levels of intensity. As a consequence of flow limitation, to cope with the increased ventilatory demands of exercise, these patients show a breathing pattern that ultimately places greater demands on their inspiratory muscles. Indeed, in these patients a pattern of low $V \mathrm{~T}$ and high frequency breathing is unlikely to be advantageous but is the only pattern available in the presence of expiratory flow limitation and dynamic hyperinflation. Increased inspiratory muscle work may contribute to dyspnoea and exercise limitation in such patients even before their ventilatory ceiling is attained.

How does NPPV work? In recent years there has been an increasing interest in the use of noninvasive positive pressure ventilation (NPPV) to increase exercise capacity of these patients. Reducing exercise dyspnoea, by unloading respiratory muscles by assisted ventilation, might allow for higher levels of exercise intensity. NPPV has been used in physiological studies in normals. By unloading respiratory muscles by means of proportional assist ventilation (PAV), a recent modality of mechanical ventilation [31], it has been shown in healthy trained cyclists that work of breathing (WOB) needed to sustain heavy-intensity exercise was correlated to a reduction in leg blood flow and had a significant influence on exercise performance $[32,33]$. In other words there would be a competition for blood flow between respiratory and limb muscles. In another study PAV prevented exercise-induced diaphragmatic fatigue as determined by bilateral phrenic nerve stimulation at all frequencies and times post-exercise [34]. To the extent that intrinsic mechanical loading and functional inspiratory muscle weakness in COPD contribute to dyspnoea, assisted ventilation should provide a symptomatic benefit by unloading and assisting such overburdened ventilatory muscles. Several studies have examined the acute effects of different modalities of ventilatory assistance on dyspnoea and exercise tolerance in advanced COPD [35].

Continuous positive airway pressure. Theoretically, continuous positive airway pressure (CPAP) should reduce the ITL on the inspiratory muscles of hyperinflated COPD patients and enhance neuromuscular coupling, thus improving dyspnoea and exercise tolerance [36]. In a study by O'DONNELL et al. [37] in COPD patients (mean forced expiratory volume in one second (FEV1) 35\% predicted) CPAP of $4-5 \mathrm{cmH}_{2} \mathrm{O}$ resulted in a significant increase in exercise endurance time. In another study these authors administered CPAP, 4-5 $\mathrm{cmH}_{2} \mathrm{O}$ during steady-state submaximal exercise in COPD patients (mean FEV1 40\% pred) and in healthy subjects [38]. CPAP, when administered to COPD patients, resulted in a highly significant reduction in the sense of breathing effort. In contrast, CPAP significantly increased the sense of breathing effort in the normal group. In a study of COPD patients (FEV1 25\% pred) CPAP reduced inspiratory muscle effort, as indicated by the transdiaphragmatic and oesophageal pressure-time integrals [39].

The commonly accepted explanation of the effects of CPAP is that it counterbalances PEEPi, i.e. the ITL [13]. From the 
above studies it can be also argued that for maximal benefit CPAP should be titrated on an individual basis to optimise comfort at pressure levels just below the ITL. Nevertheless, this should theoretically imply measurement of PEEPi, which is rather difficult to perform on a routine basis. CPAP was also shown to reduce the WOB and increase exercise tolerance in patients with cystic fibrosis. These beneficial effects were related to disease severity [40].

Pressure support ventilation. Inspiratory pressure support (IPS) is a form of mechanical ventilation that can effectively assist ventilation when applied noninvasively to patients in acute and chronic respiratory failure. Pressure support is a pressure-targeted mode in which each breath is patient triggered and supported [41]. It provides breathby-breath ventilatory support by means of a positive pressure wave synchronised with the inspiratory effort of the patient. During inspiration, the airway pressure is raised to a preset level: the pressure support level. This level is maintained until the machine determines the end of a patient's respiratory effort or detects a patient's demand for expiration. The expiratory phase is free of assistance and a level of external PEEP, lower than the inspiratory plateau pressure can be applied [41].

The excessive load placed on inspiratory muscles when patients with COPD exercise could lead to fatigue and contribute to exercise limitation. Slowing of maximal relaxation rate (MRR) of skeletal muscle is an early index of the fatiguing process. KYROUSSIS et al. [42] found in COPD patients (mean FEV1 28\% pred) walking to exhaustion a postexercise fall in oesophageal MRR, indicating that the inspiratory muscles of these patients were sufficiently heavily loaded to initiate the fatiguing process. The same authors gave a physiological confirmation of this hypothesis through an application of NPPV to unload inspiratory muscles during exercise in COPD patients [43]. They compared oesophageal MRR after equidistant treadmill walking. After a free walk there was a mean $41 \%$ slowing of oesophageal MRR. After noninvasive IPS assisted walks, the slowing of oesophageal MRR was significantly less than after the free walk.

KEILTY et al. [44] studied COPD patients with disabling breathlessness (mean FEV1 0.73 L). Studies were performed with mask IPS (mean airway pressure $12-15 \mathrm{cmH}_{2} \mathrm{O}$ ), CPAP $6 \mathrm{cmH}_{2} \mathrm{O}$, and with oxygen $\left(2 \mathrm{~L} \cdot \mathrm{min}^{-1}\right.$ via the mask) in random order on three separate days. Each of these walks was compared with a control walk using a sham circuit (breathing air via a mask at $2 \mathrm{~L} \cdot \mathrm{min}^{-1}$ from an unlabelled cylinder), and with a baseline walk in which patients walked freely on the treadmill. No patients stopped due to leg fatigue, all stopping only when their sensation of breathlessness had reached level 5 on the Borg scale [45]. NPPV improved median walking distance by $62 \%$ compared with the control walk (sham circuit). There was no change in walking distance with either CPAP or oxygen at $2 \mathrm{~L} \cdot \mathrm{min}^{-1}$. There was no difference between the control and the baseline walks. In a study by MaltAis et al. [46] severe COPD patients (mean FEV1 $0.75 \mathrm{~L})$ performed constant workload bicycle exercise during control conditions and with the application of IPS $\left(\sim 10 \mathrm{cmH}_{2} \mathrm{O}\right)$. IPS increased minute ventilation as a result of changes in both $V \mathrm{~T}$ and respiratory rate. This occurred despite marked reductions in inspiratory effort, as indicated by the oesophageal and transdiaphragmatic pressure-time integrals. Breathlessness improved significantly with the addition of IPS and worsened to a similar degree when it was removed. In another study during exhaustive treadmill walking in patients with severe COPD, the oesophageal and transdiaphragmatic pressure/time products rose early in the walk and then remained at that level until the patients stopped because of intolerable dyspnoea [47]. When patients walked the same distance assisted by IPS, a substantial reduction was observed in the inspiratory and expiratory pressure/time products throughout the walk. When patients walked with NPPV for as long as they could, the pressure/time products observed at exercise cessation were lower than those observed during exercise cessation after free walking. It was concluded that, in severe COPD, inspiratory muscle pressure generation did not increase to meet the demands imposed by exhaustive exercise.

Plasma lactate levels were measured in patients with severe COPD who performed two treadmill walks at an identical constant work rate to a condition of severe dyspnoea; the second walk was supported by IPS [48]. Lactate levels significantly increased during both walks to similar levels respectively, but the duration of the IPS-assisted walk was significantly greater than the free walk (13.6 versus $5.5 \mathrm{~min}$ ). Therefore, it was argued that patients with severe COPD can sustain exercise-induced lactataemia for longer if assisted with IPS. Another more recent study has shown that in contrast to $5 \mathrm{cmH}_{2} \mathrm{O}$, the application of IPS of $10 \mathrm{cmH}_{2} \mathrm{O}$ during exercise resulted in a statistically significant improvement in exercise endurance in patients with COPD, compared with exercise without IPS [49]. However, on an individual basis, large differences in responses were found.

Proportional assisted ventilation. Proportional assisted ventilation is a mode of partial ventilatory assistance endowed with characteristics of proportionality and adaptability to the intensity and timing of spontaneous ventilatory pattern by providing inspiratory flow and pressure in proportion to the patient's effort. The ventilator delivers pressure according to the motion equation, generating pressure in proportion to a patient's spontaneous effort. A portion of the total mechanical workload, i.e. elastance and resistance, is taken over according to a level of assistance, which has been decided by the caregiver and can specifically unload the resistive burden (flow assist) and the elastic burden (volume assist) [33, 50-52]. Dolmage and Goldstein [53] determined whether PAV applied during constant power submaximal exercise could enable severe, stable COPD patients (mean FEV $129 \%$ pred) to increase their exercise tolerance. Patients completed five sessions of cycling at $60-70 \%$ of their maximum power. The sessions differed only in the type of inspiratory assist: 1) baseline; 2) PAV; 3 ) CPAP $\left(5 \pm 2 \mathrm{cmH}_{2} \mathrm{O}\right)$; (4) PAV and CPAP; and 5) sham. Subjects reached the same level of dyspnoea (by Borg scale) during all sessions but only PAV and CPAP significantly increased exercise tolerance $(12.9 \mathrm{~min})$ versus the sham session (6.60 min). Mean exercise time during the PAV and CPAP sessions was 7.10 and $8.26 \mathrm{~min}$, respectively.

BIANCHI et al. [54] investigated the impact of PAV, CPAP and IPS on exercise tolerance and breathlessness in severe stable chronically hypercapnic COPD patients. On two consecutive days, patients underwent four endurance tests on a cycle ergometer at $80 \%$ of their maximal workrate, receiving, via a nasal mask in random order, either: 1) sham ventilation (CPAP $1 \mathrm{cmH}_{2} \mathrm{O}$ ); 2) CPAP $6 \mathrm{~cm} \mathrm{H}_{2} \mathrm{O}$; 3) IPS $12-16 \mathrm{cmH}_{2} \mathrm{O}$ and PEEP $1 \mathrm{cmH}_{2} \mathrm{O}$; or 4) PAV and PEEP $1 \mathrm{cmH}_{2} \mathrm{O}$. Oxygen supply was standardised to maintain an arterial oxygen saturation of 92-93\%. In comparison with sham ventilation, PAV, PSV and CPAP were able to increase the endurance time (from 7.2 to 12,10 and $9.6 \mathrm{~min}$, respectively) and to reduce dyspnoea and oxygen flow to the nasal mask. However, the greatest improvement was observed with PAV. Other authors have confirmed the utility of PAV to improve exercise tolerance in COPD patients [55]. 
Controlled mechanical ventilation. Assist-control ventilation is a combination mode of ventilation in which the ventilator delivers a positive pressure breath at a preset $V \mathrm{~T}$ in response to the patient's inspiratory effort. The ventilator will also deliver breaths at a preset rate if no patient effort occurs within the preselected time period [56]. Assist-control ventilation was delivered by means of a volume-cycled home ventilator and nasal masks during exercise in patients with pulmonary tuberculosis sequelae [57]. This modality of NPPV resulted in a significant improvement in breathlessness, and increase in exercise endurance in these patients. Nevertheless, this paper is still the only one reporting application of assist-control ventilation during exercise.

\section{NPPV and pulmonary rehabilitation}

The message of these physiological studies could be summarised as follows: acute NPPV, either CPAP, IPS or PAV, during exercise reduces dyspnoea and WOB and enhances exercise tolerance in COPD patients. A systematic review by VAN'T HUL et al. [58] identified 15 physiological studies dealing with use of NPPV during exercise. Seven of these studies met the inclusion criteria, including a total of 65 patients with COPD. The methodological quality of the included studies varied from $31-54 \%$ of the maximum score of 13 points. Statistically significant summary effect sizes were found in the analysis of exertional dyspnoea as well as in the analysis of exercise endurance, indicating improvements in these outcomes in favour of NPPV. Nevertheless, the role of NPPV in pulmonary rehabilitation, if any, is still to be defined. In fact, the recent development of new therapeutic approaches like lung transplantation and lung volume reduction surgery make patients with severe COPD, even with chronic respiratory failure, candidates to rehabilitation programmes [59, 60].

BIANCHI et al. [61] evaluated the effects of the addition of assisted ventilation during exercise training on the outcome of a structured pulmonary rehabilitation programme in COPD patients. Patients with stable COPD (mean FEV1 44\% pred), without chronic ventilatory failure, undergoing a 6-week multidisciplinary outpatient pulmonary rehabilitation programme including exercise training, were randomised to training during either mask PAV or spontaneous breathing. Five of 18 patients $(28 \%)$ in the PAV group but no patient in the control group dropped out due to lack of compliance with the equipment. Both groups showed significant post training improvements in exercise tolerance (peak work rate difference: 20 and 14 watts in PAV and control group, respectively), dyspnoea and leg fatigue, but not in HRQL, without any significant difference between groups. HAwKINS et al. [62] evaluated the effects of providing ventilatory assistance to patients with more severe COPD (mean FEV1 27\% pred) during a 6-week supervised high intensity outpatient cycle exercise programme. Patients were randomised either to exercise with ventilatory assistance using PAV or to exercise unaided. Before and after training, patients performed a maximal symptom limited incremental cycle test to determine peak work rate followed by a constant work rate test at $70 \%$ of peak work rate achieved in the baseline incremental test. At 6 weeks mean training intensity and peak work rate were $15.2 \%$ and $18.4 \%$ higher in the group that used PAV. Isoworkload lactataemia after training was reduced by $30 \%$ in the assisted group and by $11 \%$ in the unassisted group. A significant inverse relationship was found between reduction in iso-workload lactataemia after training during the constant work rate test and peak work rate achieved during the last week of training. The authors suggest that the reduction in plasma lactate concentration for a given workload and the correlation between the reduction in plasma lactate concentration and the increase in peak work rate in the PAV group of the study by Hawkins et al. [62] is a marker of true physiological training effect. Other authors have confirmed benefits of addition of NPPV to pulmonary rehabilitation that were not observed with Heliox breathing [63, 64].

Why does NPPV sometimes fail? The study by BIANCHI et al. [61] found no additional benefit of assisted ventilation on exercise tolerance, dyspnoea and health status when compared with training alone. Moreover, the high rate of dropouts due to lack of compliance in the PAV group and the time spent to set the ventilator and to supervise the training session during assisted ventilation were practical drawbacks of the addition of mask PAV during a high intensity training programme, at least in that setting and in those patients with moderately severe stable COPD. Those results indicate it is not worthwhile to submit patients to unpleasant equipment (e.g. mask and related troubles), with the need of constant supervision of an individual operator to check for leaks, to reset the ventilator when needed and a substantial risk of lack of compliance. A potential complication of PAV is that its appropriate setting requires measurement of the patient's respiratory mechanics. In the study by BIANCHI et al. [61] PAV was set to the patient's comfort without any measurement of respiratory mechanics that otherwise would be impossible in a routine setting. In a study conducted in stable, chronically hypercapnic COPD patients, mask PAV, set at the patient's comfort, was able to unload $\sim 70 \%$ of the total elastic load of the respiratory system but only $26 \%$ of the resistive burden [51]. Furthermore, it should be considered that patients' respiratory mechanics can change during exercise, for example due to increase of PEEPi [39], thus profoundly affecting the effect of NPPV. A change in PEEPi may affect the observed influence of PAV because PAV does not compensate for PEEPi. PAV, or any other inspiratory pressure assist, is not initiated until PEEPi is overcome, which is why exercise improves when PAV is superimposed upon CPAP. CPAP compensates for the threshold load while PAV compensates the elastic and resistive load of inspiration. Nevertheless, to explain the "negative" results of the study by BIANCHI et al. [61], the ventilatory demands of the exercising patients relative to the capacity of the ventilator must also be considered. Commercial ventilators often cannot accommodate the demand of an exercising COPD patient, which is why they may not comply. Furthermore, PAV during training may have limited role in the general population of COPD patients, many of whom have significant comorbidity.

Night-time NPPV and day-time exercise. There is another way to use NPPV to improve results of pulmonary rehabilitation. Patients with severe stable COPD were randomised by GARROD et al. [65] to home nocturnal NPPV and daytime exercise training or exercise training alone. After an 8-week training programme there was a significant improvement in mean shuttle walk test in the NPPV and exercise training group compared with the control group. The differences between the two groups became evident only in the final 4 weeks of the training programme. There was a significant improvement in HRQL. Only the NPPV group demonstrated a significant improvement in arterial oxygenation. Nocturnal NPPV is not necessarily directed at exercise and peripheral muscle adaptation. The main purpose is to correct night-time blood gases with a carryover through the day. The result should be an improvement in function including the ability to complete a daily exercise programme. This may be analogous to optimal pharmacological therapy. 
Indeed the hypothesis that the observed increased exercise performance with nocturnal NPPV may be associated with increased quadriceps strength was not confirmed [66]. Quadriceps strength was measured as the twitch tension elicited by magnetic stimulation of the femoral nerve and the maximum voluntary contraction force in patients with chronic respiratory failure due to $\mathrm{COPD}$ and restrictive thoracic disease before and after 2-month NPPV. After NPPV there was significant clinical, arterial blood gas and walking distance improvement, but there was no improvement in quadriceps strength [66].

\section{Methodological considerations}

Any conclusion about the potential clinical utility of ventilatory assistance during exercise is difficult because of small study sample size, variability of pathophysiological abnormalities at study entry, differences in the ventilatory devices and operating characteristics of the ventilators, differences in pressure optimisation protocols, or lack of optimisation, differences in breathing circuit design with the potential for carbon dioxide rebreathing and differences in exercise protocols and evaluative methods of dyspnoea. Larger prospective controlled studies should be required to determine if ventilatory assistance will eventually be a useful adjunct to standard exercise protocols for dyspnoeic patients with more advanced cardiopulmonary diseases. The alternative is well-designed small samples regardless of positive or negative results. Replication of these studies will help guard against type I error. By replicating or repeating any experiment that is conducted with a "relaxed" significance level, the authors should be able to protect themselves from reporting a type I error while maintaining reasonable statistical power and sensitivity in an initial exploratory investigation. As in any study, large or small, a thorough description of subjects is required; the ability of the ventilatory device to meet the demands of the patient must be demonstrated; the operating characteristics described and carbon dioxide rebreathing must be within acceptable limits.

\section{Electrical stimulation}

Neuromuscular electrical stimulation (NMES) is used by physical therapists to improve muscle performance. This type of stimulation is characterised by a low-volt stimulation targeted to stimulate motor nerves to cause a muscle contraction. NMES has been used to increase healthy muscles performance [67, 68]. Application of NMES has been consistently associated with increased mass, strength, and endurance of both normally and abnormally innervated muscles in a range of pathological conditions [69-71]. In fact NMES retards muscle wasting during denervation immobilisation and optimises recovery of muscle strength during rehabilitation. It has been also used to assist respiratory function in patients with spinal cord injury [71-73]. There is growing evidence that NMES can be safely and effectively used in patients with skeletal muscle function deficit and exercise intolerance due to systemic diseases [74-77]. Low-frequency muscular electrical stimulation has been shown to induce an increase in the muscular oxidative capacities and could represent another form of mild physical training. It was well tolerated and induced an increased exercise capacity in patients with congestive heart failure, without undesirable increases in cardiac output. This form of training can be achieved at home and is local or loco-regional so it could be expected that it would not induce any adverse acute cardiac side-effect or late remodelling [74]. NMES treatment of thigh muscles using a combined NMES protocol comprising biphasic, symmetric, rectangular constant current impulses at different frequencies and amplitudes to enhance strength and endurance capacity appeared to be safe even in patients with heart failure and implanted pacemakers with bipolar sensing [78].

NMES and COPD. Pulmonary rehabilitation in active COPD patients may normalise the electrical activity of skeletal muscles during incremental dynamic exercise. The electromyographical signal confirms neuromuscular changes after endurance training [79]. Passive training of specific locomotor muscle groups by means of NMES might be better tolerated than whole body exercise in patients with severe COPD. It was hypothesised that this novel strategy would be particularly effective in improving functional impairment and the consequent disability that characterises patients with end stage COPD. Three small controlled studies of this technique in severe COPD patients have been reported [80-82]. Patients (mean FEV1 38\% pred) with advanced COPD were randomly assigned to either a home based 6-week quadriceps femoris NMES training programme or a 6 -week control period before receiving NMES [80]. All patients were able to complete the NMES training programme successfully, even in the presence of exacerbations. Training was associated with significant improvements in muscle function, maximal and endurance exercise tolerance, and the dyspnoea domain of the Chronic Respiratory Disease Questionnaire [83]. Improvements in muscle performance and exercise capacity after NMES correlated well with reduction in perception of leg effort corrected for exercise intensity. Another randomised controlled trial of transcutaneous electrical muscle stimulation of the lower extremities was performed in stable patients (mean FEV1 38\% pred) [81]. Stimulation of the lower extremities was performed three times a week, 20 min each session, for six continuous weeks. Electrical muscle stimulation improved both the quadriceps strength $(39.0 \%$ versus $9.0 \%)$ and hamstring muscle strength $(33.9 \%$ versus $2.9 \%)$ in the treated and sham treated groups, respectively. The improvement in muscle strength carried over to better performance in the shuttle walk test in the treated group (36.1\% versus $1.6 \%$ in the treated and sham groups respectively). There was no significant change in lung function, peak workload, or peak oxygen consumption in either group. Muscle stimulation was well tolerated by the patients with no dropouts and had $>95 \%$ compliance with the protocol. NMES has also been applied in bed-bound COPD patients receiving mechanical ventilation with marked peripheral muscle hypotonia and atrophy. ZANOTTI et al. [82] compared the effects of active limb mobilisation with or without NMES on muscle strength, respiratory rate, heart rate, oxygen saturation, and time needed to transfer from bed to chair in two groups of bed-bound patients with chronic hypercapnic respiratory failure due to COPD who were receiving mechanical ventilation, with marked peripheral muscle hypotonia and atrophy. Patients were randomly assigned either to active limb mobilisation alone or to active limb mobilisation plus NMES. NMES was applied using square-wave alternate, symmetric, and compensated impulses for $30 \mathrm{~min}$ b.i.d. The duration of treatment was 28 days for all patients. Muscle strength improved significantly in the overall group of patients. Addition of NMES resulted in significantly improved muscle strength and respiratory rate and decreased the number of days needed to transfer from bed to chair. The major advantage of NMES over conventional exercise training is considered to be the lack of ventilatory stress during passive muscular activity, reflecting the reduced muscle mass involved. 
Furthermore, one of these studies [80] indicates that NMES may be used even during periods of exacerbations, a condition associated with loss of muscle strength and mass [84].

However, there are negative studies in which no significant change in neurophysiological, aerobic performance or clinical status occurred after electrostimulation $[85,86]$. Furthermore, studies in COPD may have limitations: e.g. a sham/placebo was not included in the study by NEDER et al. [80], patients observed are limited, therefore these studies must be considered as preliminary, and NMES should be still considered as an experimental tool in pulmonary rehabilitation. Further studies are necessary to include this modality in routine pulmonary rehabilitation programmes. In particular, further randomised trials using larger samples and longer follow-up periods are needed. Optimal forms of stimulation settings are yet to be determined, as are possible sex-related differences in responsiveness to electrical stimulation. For example, in a study in normal subjects muscle torque and fatigue of electrically induced contractions depended on the waveform used to stimulate the contraction, with monophasic and biphasic waveforms having an advantage over the polyphasic waveform [87]. Morphological studies correlating improvements in exercise tolerance with muscular changes described with conventional exercise training are also needed $[25,26]$.

\section{Interval training}

Interval training is characterised by repeated bouts of highintensity exercise interspersed with recovery periods (light exercise or rest) [88]. In healthy subjects, interval training with rest during the recovery period has been widely studied and, in some studies, has shown better results than continuous training [88-93]. Gorostiaga et al. [89] showed a greater increase in peak oxygen consumption and peak work rate after an interval training programme than after continuous training. GAESSER and WILSON [90] showed that peak oxygen consumption increased significantly only with interval training and PoOLE and GAESSER [91] showed that the lactate threshold exhibited greater improvements in interval training than in continuous training. AMAIDI et al. [92] found that, in elderly people, interval training was more easily accepted and tolerated than continuous training. Theoretical explanations for superior responses to interval training have been proposed. High intensity work rate for 1 or 2 min induces high blood lactate levels due to the depletion of phosphocreatine and the use of oxygen myoglobin-bound reserves, but interspersed periods of sub-lactate threshold work rates may facilitate lactate removal [88]. When compared with the same total amount of work performed continuously, interval training has been shown to induce less lactate accumulation [93] and to prevent glycogen depletion by favouring the metabolism of lipids [88].

Interval training in COPD. In patients with COPD, interval training has been shown capable of inducing physiological training effects $[14,94-100]$. The most common interval training protocol studied in these patients is moderate to high intensity exercise alternated with low-intensity exercise [96, 98-102]. Rehabilitation programmes, including interval training, resulted in delay in lactate threshold, increase in peak work rate and peak oxygen consumption and improvements in HRQL [96, 98]. After an interval training programme, an increase in peak oxygen extraction ratio, reduced phosphocreatine recovery time and improved cellular bioenergetics have been shown [101]. Nevertheless, the superiority of high-intensity bilevel interval over moderate-intensity continuous training was not clearly demonstrated by COPPOOLSE et al. [97].

\section{Oxygen supplementation}

Supplemental oxygen has the potential to increase exercise tolerance of the hypoxaemic COPD patient by different mechanisms: hypoxic stimulation of the carotid bodies is reduced, the pulmonary circulation vasodilates and arterial oxygen content increases. The latter two mechanisms may potentially reduce carotid body stimulation at heavy levels of exercise by increasing oxygen delivery to the exercising muscles and reducing carotid body stimulation by lactic acidaemia. The main mechanism for oxygen's effect on exercise tolerance has recently been clarified [103]. Ambulatory oxygen therapy has widely been shown to increase exercise performance and to relieve exercise breathlessness in COPD patients [104-108]. Recent studies indicate that reduction in hyperinflation plays an important role in the oxygen-related relief of dyspnoea [104, 108]. Interestingly, supplemental oxygen generally increases exercise tolerance in patients with only mild to moderate hypoxaemia (i.e. levels of hypoxaemia not severe enough to meet guidelines for LTOT) [104, 109, 110].

Despite these positive physiological results, a study by TERrADos et al. [111], suggests that mild hypoxaemia accelerates peripheral muscle adaptation such that the use of supplemental oxygen during training of mildly hypoxaemic patients may not be advantageous. This seems to have been confirmed by previous studies of COPD patients trained while using supplemental oxygen. These studies failed to demonstrate benefits of supplemental oxygen during rehabilitation [112-116]. A recently published double blind study involved nonhypoxaemic patients with severe COPD (FEV1 36\% pred) exercised in a 7 week outpatient programme [117]. During exercise they received by nasal cannula either oxygen $\left(3 \mathrm{~L} \cdot \mathrm{min}^{-1}\right)$ or compressed air $\left(3 \mathrm{~L} \cdot \mathrm{min}^{-1}\right)$. Exercise was on cycle ergometers for $45 \mathrm{~min}, 3$ times per week; as the programme proceeded work rate was progressively increased. Both groups had higher exercise tolerance (while breathing air) after training. However, the oxygen trained group increased training intensity more rapidly over the 7 weeks than the control group. After training, exercise endurance increased significantly more in the oxygen trained group $(213 \%)$ than in controls $(170 \%)$. This study [117] differed somehow from others [112-116]. Indeed in this study [117] sufficient supplemental oxygen was given during training to raise arterial oxygen saturation, subjects were urged to maximise their training work rates so that any increase in exercise tolerance produced by oxygen breathing would result in higher training intensity and both effort dependent and effort independent measures of exercise tolerance were utilised to detect the magnitude of the training effect. Nevertheless, other studies have achieved increases in oxygen uptake with high intensity training without supplemental oxygen [118]. Further studies are needed to define the appropriate patients in order to generalise such intervention. In the light of the present knowledge oxygen supplementation during training should be limited to individual cases.

\section{Biofeedback}

Biofeedback refers to techniques to teach self-control over physiological functions. In other words people might be taught to increase or decrease the activity of internal bodily functions. The most common application of biofeedback has 
been stress management by teaching major muscle groups relaxation. More recently using biofeedback patients have tried to modify such autonomic functions as vasomotor activity, heart rate, blood pressure, and bronchomotor tone. Several biofeedback techniques have been applied to patients with pulmonary disorders in a frame of pulmonary rehabilitation programmes. In respiratory biofeedback a signal relating to some respiratory function is monitored and displayed to the patient, who tries to modify that function. Biofeedback has been used in patients with asthma, emphysema, and pulmonary fibrosis and has been used to improve gas exchange and reduce the WOB [119]. Techniques varied from relaxation and stress management to patient selfcontrol. Electromyography, incentive spirometry, airway resistance signals and pulse oximetry have been used [119, 120]. Training the patient in pursed-lips breathing guided by oximetry, and decreasing the time of weaning from mechanical ventilation are examples of biofeedback application to respiratory patients [121, 122]. Application of biofeedback to exercise in COPD patients has been less studied. In a study by CoLlins et al. [123] to determine the effects of biofeedback during exercise training of COPD, the authors concluded that exercise training with biofeedback and exercise training alone were equally effective in improving leg-cycle exercise tolerance in patients with moderate-to-severe COPD. However, the inability to conclude an improvement with biofeedback combined with exercise training over exercise training alone may have been due to lack of statistical power. Clearly this issue does require further investigation.

\section{Final considerations}

The positive conclusion from the above studies is that multidisciplinary pulmonary rehabilitation in its consolidated "old" modalities, as evaluated by evidence based medicine is a valuable adjunct to treatment of chronic obstructive pulmonary disease $[3,5,8-11]$. In this frame exercise training is a cornerstone. The value of studies supporting the use of these newer strategies in the clinical management of chronic obstructive pulmonary disease patients differs substantially among these techniques. Indeed, clinical trials designed to establish that a given intervention yields superior results compared with standard interventions are generally difficult to perform because of the rather wide variability of responses to exercise interventions among chronic obstructive pulmonary disease patients. While high intensity training may be useful to induce a true physiological response to training, it does not necessarily translate into better health-related quality of life compared with low intensity training. Nevertheless, this does not mean that patients would not have benefited from a more intense exercise programme and improved cardiopulmonary capacity. Therefore, there is a theoretical reason to go on with research in tools to increase exercise intensity patients can tolerate. Furthermore, the modalities discussed should be used as adjuncts to a welldesigned comprehensive respiratory rehabilitation programme including other interventions, such as education, nutrition, and psychological counselling.

\section{References}

1. Report of British Research Medical Council Working Party. Long-term domiciliary oxygen therapy in chronic hypoxic cor pulmonale complication in chronic bronchitis and emphysema. Lancet 1981; 1: 681-686.

2. Anthonisen NR, Connett JE, Kiley JP, et al. Effects of smoking intervention and the use of an inhaled anticholinergic bronchodilator on the rate of decline of FEV1. The Lung Health Study. JAMA 1994; 272: 14971505.

3. Pauwels RA, Buist AS, Calverley PMA, Jenkins CR, Hurd SS on behalf of the GOLD Scientific Committee. Global strategy for the diagnosis, management and prevention of chronic obstructive lung disease. NHLBI/WHO global initiative for chronic obstructive lung disease (GOLD) workshop summary. Am J Respir Crit Care Med 2001; 163 : 1256-1276.

4. Restrick LJ, Paul EA, Braid GM, Cullinan P, Moore-Gillon J, Wedzicha JA. Assessment and follow up of patients prescribed long-term oxygen therapy. Thorax 1993; 48: 708713.

5. Donner CF, Decramer M. Pulmonary rehabilitation. Eur Respir Mon 2000; 5: 1-199.

6. Decramer M, Gosselink R, Trooster T, Verschueren M, Evers G. Muscle weakness is related to utilization of healthcare resources in COPD patients. Eur Respir J 1997; 10: $417-423$.

7. Connors AF jr, Dawson NV, Thomas C, et al. Outcomes following acute exacerbations of severe chronic obstructive lung disease. The SUPPORT investigators. Am J Respir Crit Care Med 1996; 154: 959-967.

8. Donner CF, Howard P. Pulmonary rehabilitation in chronic obstructive pulmonary disease (COPD) with recommendations for its use. Eur Respir J 1992; 5: 266-275.

9. NIH Workshop Summary: Pulmonary Rehabilitation Research. Am J Respir Crit Care Med 1994; 149: 825-833.

10. American Thoracic Society. Pulmonary rehabilitation 1999. Am J Respir Crit Care Med 1999; 159: 1666-1668.

11. British Thoracic Society. Standards of care subcommittee on pulmonary rehabilitation. Thorax 2001; 56: 827-834.

12. Rossi A, Polese G, Brandi G, Conti G. Intrinsic positive endexpiratory pressure (PEEPi). Intensive Care Med 1995; 21: $522-536$

13. $\mathrm{O}^{\prime}$ Donnell DE. Exertional breathlessness in chronic respiratory disease. In: DA Mahler, ed. Dyspnea. New York, Dekker, 1998; pp. 97-147.

14. American Thoracic Society/European Respiratory Society. Skeletal muscle dysfunction in chronic obstructive pulmonary disease. Am J Respir Crit Care Med 1999; 159: S1-S40.

15. Schols AMWJ, Slangen J, Volovics L, Wouters EFM. Weight loss is a reversible factor in the prognosis of chronic obstructive pulmonary disease. Am J Respir Crit Care Med 1998; 157: 1791-1797.

16. Musaro A, McCullagh K, Paul A, Houghton L, et al. Localized Igf-1 transgene expression sustains hypertrophy and regeneration in senescent skeletal muscle. Nat Genet 2001; 27: 195-200.

17. Debigarè $\mathrm{R}$, Còtè $\mathrm{CH}$, Maltais $\mathrm{F}$. Peripheral muscle wasting in chronic obstructive pulmonary disease. Clinical relevance and mechanisms. Am J Respir Crit Care Med 2001; 164: 1712-1717.

18. Schols AMWJ, Soeters PB, Mostert R, Pluymers RJ, Wouters EFM. Physiologic effects of nutritional support and anabolic steroids in patients with chronic obstructive pulmonary disease. Am J Respir Crit Care Med 1995; 152: $1268-1274$.

19. Burdet L, de Muralt B, Schutz Y, Pichard C, Fitting JW. Administration of growth hormone to underweight patients with chronic obstructive pulmonary disease. A prospective randomized, controlled study. Am J Respir Crit Care Med 1997; 156: 1800-1806.

20. Willoughby DS, Priest JW, Jennings RA. Myosin heavy chain isoform and ubiquitin protease mRNA expression after passive leg cycling in persons with spinal cord injury. Arch Phys Med Rehabil 2000; 81: 157-163.

21. Rabinovich RA, Ardite E, Troosters T, et al. Reduced muscle redox capacity after endurance training in patients with chronic obstructive pulmonary disease. Am J Respir Crit Care Med 2001; 164: 1114-1118. 
22. Casaburi R, Patessio A, Ioli F, Zanaboni S, Donner CF, Wasserman K. Reduction in exercise lactic acidosis and ventilation as a result of exercise training in patients with obstructive lung disease. Am Rev Respir Dis 1991; 143: 9-18.

23. Casaburi R, Porszasz J, Burns MR, Carithers ER, Chang RSY, Cooper CB. Physiologic benefits of exercise training in rehabilitation of severe COPD patients. Am J Respir Crit Care Med 1997; 155: 1541-1551.

24. Casaburi R. Mechanisms of the reduced ventilatory requirement as a result of exercise training. Eur Respir Rev 1995; 5: 25, 42-46.

25. Maltais F, LeBlanc P, Simard C, et al. Skeletal muscle adaptation to endurance training in patients with chronic obstructive pulmonary disease. Am J Respir Crit Care Med 1996; 154: 442-447.

26. Jobin J, Maltais F, Doyon JF, et al. Chronic obstructive pulmonary disease: capillarity and fiber-type characteristics of skeletal muscle. J Cardiopulm Rehabil 1998; 18: 432-437.

27. Lacasse Y, Wong E, Guyatt GH, Cook DJ, Goldstein RS. Meta-analysis of respiratory rehabilitation in chronic obstructive pulmonary disease. Lancet 1996; 348: 1115-1119.

28. ACCP/AACVPR. Pulmonary rehabilitation. Joint ACCP/ AACVPR evidence-based guidelines. Chest 1997; 112: 13631396.

29. Gosselink R, Troosters T, Decramer M. Exercise training in COPD patients: the basic questions. Eur Respir $J$ 1997; 10 : 2884-2891.

30. Maltais F, LeBlanc $\mathrm{P}$, Jobin $\mathrm{J}$, et al. Intensity of training and physiologic adaptation in patients with chronic obstructive pulmonary disease. Am J Respir Crit Care Med 1997; 155: $555-561$.

31. Younes M. Proportional assist ventilation. In: Tobin MJ ed. Principles and practice of mechanical ventilation. New York, McGraw-Hill inc, 1994; pp. 349-370.

32. Harms CA, Babcock MA, McClaran SR, et al. Respiratory muscle work compromises leg blood flow during maximal exercise. J Appl Physiol 1997; 82: 1573-1583.

33. Harms CA, Wetter TJ, St Croix CM, Pegelow DF, Dempsey JA. Effects of respiratory muscle work on exercise performance. J Appl Physiol 2000; 89: 131-138.

34. Babcock MA, Pegelow DF, Harms CA, Dempsey JA. Effects of respiratory muscle unloading on exercise-induced diaphragm fatigue. J Appl Physiol 2002; 93: 201-206.

35. Ambrosino N. Exercise and noninvasive ventilatory support. Monaldi Arch Chest Dis 2000; 55: 242-246.

36. Lougheed MD, Webb KA, O'Donnell DE. Breathlessness during induced hyperinflation in asthma: role of the inspiratory threshold load. Am J Respir Crit Care Med 1995; 152: 911-920.

37. O'Donnell DE, Sanii R, Younes M. Improvement in exercise endurance in patients with chronic airflow. Limitation using continuous positive airway pressure. Am Rev Respir Dis 1988; 138: 1510-1514.

38. O'Donnell DE, Sanii R, Giesbrecht G, Younes M. Effect of continuous positive airway pressure on respiratory sensation in patients with chronic obstructive pulmonary disease during submaximal exercise. Am Rev Respir Dis 1988; 138: 1185-1191.

39. Petrof BJ, Calderini E, Gottfried SB. Effect of CPAP on respiratory effort and dyspnoea during exercise in severe COPD. J Appl Physiol 1990; 69: 179-188.

40. Henke KG, Regnis JA, Bye PTP. Benefits of continuous positive airway pressure during exercise in cystic fibrosis and relationship to disease severity. Am Rev Respir Dis 1993; 148: 1272-1276.

41. Brochard L. Pressure support ventilation. In: Tobin MJ ed. Principles and practice of mechanical ventilation. New York, McGraw-Hill inc, 1994; pp. 239-256.

42. Kyroussis D, Polkey MI, Keilty SE, et al. Exhaustive exercise slows inspiratory muscle relaxation rate in chronic obstructive pulmonary disease. Am J Respir Crit Care Med 1996; 153: 787-793.
43. Polkey MI, Kyroussis D, Mills GH, et al. Inspiratory pressure support reduces slowing of inspiratory muscle relaxation rate during exhaustive treadmill walking in severe COPD. Am J Respir Crit Care Med 1996; 154: $1146-1150$

44. Keilty SE, Ponte J, Fleming TA, Moxham J. Effect of inspiratory pressure support on exercise tolerance and breathlessness in patients with severe stable chronic obstructive pulmonary disease. Thorax 1994; 49: 990-996.

45. Borg GAV. Psycophysical basis of perceived exertion. Med Sci Sports Exerc 1992; 14: 377-381.

46. Maltais F, Reissmann H, Gottfried SB. Pressure support reduces inspiratory effort and dyspnea during exercise in chronic airflow obstruction. Am J Respir Crit Care Med 1995; 151: 1027-1033.

47. Kyroussis D, Polkey MI, Hamnegard CH, Mills GH, Green M, Moxham J. Respiratory muscle activity in patients with COPD walking to exhaustion with and without pressure support. Eur Respir J 2000; 15: 649-655.

48. Polkey MI, Hawkins P, Kyroussis D, Ellum SG, Sherwood R, Moxham J. Inspiratory pressure support prolongs exercise induced lactataemia in severe COPD. Thorax 2000; 55: 547-549.

49. van't Hul A, Gosselink R, Hollander P, Postmus P, Kwakkel G. Acute effects of inspiratory pressure support during exercise in patients with COPD. Eur Respir J 2004; 23: $34-40$.

50. Ambrosino N, Vitacca M, Polese G, Pagani M, Foglio K, Rossi A. Short-term effects of nasal proportional assist ventilation in patients with chronic hypercapnic respiratory insufficiency. Eur Respir J 1997; 10: 2829-2834.

51. Polese G, Vitacca M, Bianchi L, Rossi A, Ambrosino N. Nasal proportional assist ventilation unloads the inspiratory muscles of stable patients with hypercapnia due to COPD. Eur Respir J 2000; 16: 491-498.

52. Porta R, Appendini L, Vitacca M, et al. Mask proportional assist vs pressure support ventilation in patients in clinically stable condition with chronic ventilatory failure. Chest 2002; 122: 479-488.

53. Dolmage TE, Goldstein RS. Proportional assist ventilation and exercise tolerance in subjects with COPD. Chest 1997; 111: 948-954.

54. Bianchi L, Foglio K, Pagani M, Vitacca M, Rossi A, Ambrosino N. Effects of proportional assist ventilation on exercise tolerance in COPD patients with chronic hypercapnia. Eur Resp J 1998; 11: 422-427.

55. Hernandez P, Maltais F, Gursahaney A, Leblanc P, Gottfried SB. Proportional Assist Ventilation may improve exercise performance in severe chronic obstructive pulmonary disease. J Cardiopulm Rehabil 2001; 21: 135-142.

56. Mador MJ. Assist-control ventilation. In: Tobin MJ ed. Principles and practice of mechanical ventilation. New York, McGraw-Hill inc, 1994; pp. 207-219.

57. Tsuboi T, Ohi M, Cjin K, et al. Ventilatory support during exercise in patients with pulmonary tuberculosis sequelae. Chest 1997; 112: 1000-1007.

58. van't Hul A, Kwakkel G, Gosselink R. The acute effects of noninvasive ventilatory support during exercise on exercise endurance and dyspnea in patients with chronic obstructive pulmonary disease: a systematic review. $J$ Cardiopulm Rehabil 2002; 22: 290-297.

59. Stiebellehner L, Quittan M, End A, et al. Aerobic endurance training programme improves exercise performance in lung transplant recipients. Chest 1998; 113: 906-912.

60. Criner GJ, Cordova FC, Furukawa S, et al. Prospective randomized trial comparing bilateral lung volume reduction surgery to pulmonary rehabilitation in severe chronic obstructive pulmonary disease. Am J Respir Crit Care Med 1999; 160: 2018-2027.

61. Bianchi L, Foglio K, Porta R, Baiardi R, Vitacca M, Ambrosino N. Lack of additional effect of adjunct of assisted ventilation to pulmonary rehabilitation in mild COPD patients. Respir Med 2002; 96: 359-367. 
62. Hawkins P, Johnson LC, Nikoletou D, et al. Proportional assist ventilation as an aid to exercise training in severe chronic obstructive pulmonary disease. Thorax 2002; 57: 853-859.

63. Johnson JE, Gavin DJ, Usar M, Adams-Dramiga S. Effects of training with Heliox and noninvasive positive pressure ventilation on exercise ability in patients with severe COPD. Chest 2002; 122: $464-472$.

64. Costes F, Agresti A, Court-Fortune I, Roche F, Vergnon JM, Barthelemy JC. Noninvasive ventilation during exercise training improves exercise tolerance in patients with chronic obstructive pulmonary disease. J Cardiopulm Rehabil. 2003; 23: 307-313.

65. Garrod R, Mikelsons C, Paul EA, Wedzicha JA. Randomized controlled trial of domiciliary noninvasive positive pressure ventilation and physical training in severe chronic obstructive pulmonary disease. Am J Respir Crit Care Med 2000; 162: 1335-1341.

66. Schonhofer B, Zimmermann C, Abramek P, Suchi S, Kohler D, Polkey MI. Non-invasive mechanical ventilation improves walking distance but not quadriceps strength in chronic respiratory failure. Respir Med 2003; 97: 818-824.

67. Maffiuletti NA, Cometti G, Amiridis IG, Martin A, Pousson M, Chatard JC. The effects of electromyostimulation training and basketball practice on muscle stability. Int J Sports Med 2000; 21: 437-443.

68. Hainault K, Duchateau J. Neuromuscular electrical stimulation and voluntary exercise. Sports Med 1992; 14: 100-115.

69. Lake DA. Neuromuscular electrical stimulation: an overview and its application in the treatment of sports injuries. Sports Med 1992; 13: 320-335.

70. Glaser RM. Functional neuromuscular stimulation: exercise conditioning of spinal cord injured patient. Int J Sports Med 1994; 15: 142-182.

71. Langbein WE, Maloney C, Kandare F, Stanic U, Nemchausky B, Jaeger RJ. Pulmonary function testing in spinal cord injury: effects of abdominal muscle stimulation. J Rehabil Res Dev 2001; 38: 591-597.

72. Lin VW, Singh H, Chitkara RK, Perkash I. Functional magnetic stimulation for restoring cough in patients with tetraplegia. Arch Phys Med Rehabil 1998; 79: 517-522.

73. Sorli J, Kandare F, Jaeger RJ, Stanic U. Ventilatory assistance using electrical stimulation of abdominal muscles. IEEE Trans Rehabil Eng 1996; 4: 1-6.

74. Maillefert JF, Eicher JC, Walker P, et al. Effects of lowfrequency electrical stimulation of quadriceps and calf muscles in patients with chronic heart failure. J Cardiopulm Rehabil 1998; 18: 277-282.

75. Vaquero AF, Chicharro JL, Gil L, et al. Effects of muscle electrical stimulation on peak $\mathrm{VO}_{2}$ in cardiac transplant patients. Int J Sports Med 1998; 19: 17-22.

76. Quittan M, Sochor A, Wiesinger GF, et al. Strength improvement of knee extensor muscles in patients with chronic heart failure by neuromuscular electrical stimulation. Artif Organs 1999; 23: 432-435.

77. Quittan M, Wiesinger GF, Sturm B, et al. Improvement of thigh muscles by neuromuscular electrical stimulation in patients with refractory heart failure: a single-blind, randomized, controlled trial. Am J Phys Med Rehabil 2001; 80: 206-214.

78. Crevenna R, Mayr W, Keilani M, et al. Safety of a combined strength and endurance training using neuromuscular electrical stimulation of thigh muscles in patients with heart failure and bipolar sensing cardiac pacemakers. Wien Klin Wochenschr 2003; 31: 115: 710-714.

79. Gosselin N, Lambert K, Poulain M, Martin A, Prefaut C, Varray A. Endurance training improves skeletal muscle electrical activity in active COPD patients. Muscle Nerve 2003; 28: 744-753.

80. Neder JA, Sword D, Ward SA, Mackay E, Cochrane LM, Clark CJ. Home based neuromuscular electrical stimulation as a new rehabilitative strategy for severely disabled patients with chronic obstructive pulmonary disease (COPD). Thorax 2002; 57: 333-337.

81. Bourjeily-Habr G, Rochester CL, Palermo F, Snyder P, Mohsenin V. Randomised controlled trial of transcutaneous electrical muscle stimulation of the lower extremities in patients with chronic obstructive pulmonary disease. Thorax 2002; 57: 1045-1049.

82. Zanotti E, Felicetti G, Maini M, Fracchia C. Peripheral muscle strength training in bed-bound patients with COPD receiving mechanical ventilation. Effect of electrical stimulation. Chest 2003; 124: 292-296.

83. Guyatt GH, Berman LB, Townsend M, Pugsley SO, Chambers LW. A measure of quality of life for clinical trials in chronic lung disease. Thorax 1987; 42: 773-778.

84. Engelen MP, Shols AM, Lamers RJ, Wouters E. Different patterns of chronic tissue wasting among patients with chronic obstructive pulmonary disease. Clin Nutr 1999; 18: 275-280.

85. Vengust R, Strojnik V, Pavlovic V, Antolic V, Zupanc O. The effect of electrostimulation and high load exercises in patients with patellofemoral joint dysfunction. A preliminary report. Pflugers Arch 2001; 442: Suppl. 1, 153-154.

86. Perez M, Lucia A, Rivero JL, et al. Effects of transcutaneous short-term electrical stimulation on $\mathrm{M}$. vastus lateralis characteristics of healthy young men. Pflugers Arch 2002; 443: 866-874.

87. Laufer Y, Ries JD, leininger PM, Alon G. Quadriceps femoris muscle torques and fatigue generated by neuromuscular electrical stimulation with three different waveforms. Phys Ther 2001; 81: 1307-1316.

88. Billat VL. Interval training for performance: A scientific and empirical practice. Special recommendation for middle and long distance running. Part I: Aerobic interval training. Sports Med 2001; 31: 13-31.

89. Gorostiaga EM, Walter CB, Foster C, Hickson RC. Uniqueness of interval and continuous training at the same maintained exercise intensity. Eur J Appl Physiol 1991; 63: 101-107.

90. Gaesser GA, Wilson LA. Effects of continuous and interval training on the parameters of power-endurance time relationship for high-intensity exercise. Int $J$ Sports Med 1988; 9: 417-421.

91. Poole DC, Gaesser GA. Response of ventilatory and lactate thresholds to continuous and interval trainning. $J$ Appl Physiol 1985; 58: 1115-1121.

92. Ahmaidi S, Masse-Biron J, Adam B, et al. Effects of interval training at the ventilatory threshold on clinical and cardiorespiratory responses in elderly humans. Eur $J$ Appl Physiol 1998; 78: 170-176.

93. Fox EL, Robinson S, Wiegman DL. Metabolic energy sources during continuous and interval running. $J$ Appl Physiol 1969; 27: 174-178.

94. Gimenez M, Servera E, Vergara P, Black JR, Polu JM. Endurance training in patients with chronic obstructive pulmonary disease: A comparison of high versus moderate intensity. Arch Phys Med Rehabil 2000; 81: 102-109.

95. Larson JL, Covey MK, Wirtz SE, et al. Cycle ergometer and inspiratory muscle training in chronic obstructive pulmonary disease. Am J Respir Crit Care Med 1999; 160: 500-507.

96. Vallet G, Ahmaidi S, Serres I, et al. Comparison of two training programs in chronic airflow limitation patients: standardized versus individualized protocols. Eur Respir $J$ 1997; 10: 114-122.

97. Coppoolse R, Schols AMWJ, Baarends EM, et al. Interval versus continuous training in patients with severe COPD: A randomized clinical trial. Eur Respir J 1999; 14: 258-263.

98. Fuchs-Climent D, LeGallais D, Varray A, Desplan J, Cadopi M, Préfaut C. Quality of life and exercise tolerance in chronic obstructive pulmonary disease. Effect of a short and intensive inpatient rehabilitation programme. Am J Phys Med Rehabil 1999; 78: 330-335.

99. Gosselink R, Troosters T, Decramer M. Exercise training in 
COPD patients: interval versus endurance training. Eur Respir J 1998; 12: 2s.

100. Goldstein RS, Gort EH, Stubbing D, Avendano MA, Guyatt GH. Randomised controlled trial of respiratory rehabilitation. Lancet 1994; 344: 1394-1397.

101. Sala E, Roca J, Marrades RM, et al. Effects of endurance training on skeletal muscle bioenergetics in chronic obstructive pulmonary disease. Am J Respir Crit Care Med 1999; 159: $1726-1734$.

102. Vogiatzis I, Nanas S, Roussos C. Interval training as an alternative modality to continuous exercise in patients with COPD. Eur Respir J 2002; 20: 12-19.

103. Somfay A, Porszasz J, Lee SM, Casaburi R. Effect of hyperoxia on gas exchange and lactate kinetics following exercise onset in non-hypoxemic COPD patients. Chest 2002; 121: 393-400.

104. Somfay A, Porszasz J, Lee SM, Casaburi R. Effect of oxygen on hyperinflation and exercise endurance in non-hypoxemic COPD patients. Eur Respir J 2001; 18: 77-84.

105. Stein DA, Bradley BL, Miller W. Mechanisms of oxygen effects on exercise in chronic obstructive pulmonary disease. Chest 1982; 81: 6-10.

106. Bradley BL, Garner AE, Billiu K, Mestas JM, Forma J. Oxygen-assisted exercise in chronic obstructive lung disease: the effect on exercise capacity and arterial blood gas tensions. Am Rev Respir Dis 1978; 118: 239-243.

107. Dean NC, Brown JK, Himelman RB, Doherty JJ, Gold WM, Stulbarg MS. Oxygen may improve dyspnea and endurance in patients with chronic obstructive pulmonary disease and only mild hypoxemia. Am Rev Respir Dis 1992; 148: 941-945.

108. O'Donnell DE, Bain DJ, Webb KA. Factors contributing to relief of exertional breathlessness during hyperoxia in chronic airflow limitation. Am J Respir Crit Care Med 1997; 155: 530-535.

109. Woodcock AA, Gross ER, Geddes DM. Oxygen relieves breathlessness in "pink puffers". Lancet 1981; 1: 907-909.

110. O'Donnell DE, D'Arsigny C, Webb KA. Effects of hyperoxia on ventilatory limitation during exercise in advanced chronic obstructive pulmonary disease. $\mathrm{Am}$ J Respir Crit Care Med 2001; 163: 892-898.

111. Terrados N, Jansson E, Sylven C, Kaijser L. Is hypoxia a stimulus for synthesis of oxidative enzymes and myoglobin? J Appl Physiol 1990; 68: 2369-2372.

112. Garrod R, Paul EA, Wedzicha JA. Supplemental oxygen during pulmonary rehabilitation in patients with COPD with exercise hypoxaemia. Thorax 2000; 55: 539-543.

113. Fichter J, Fleckenstein J, Stahl C, Sybrecht GW. Effect of oxygen (FI02: 0.35 ) on the aerobic capacity in patients with COPD. Pneumologie 1999; 53: 121-126.

114. McDonald CF, Blyth CM, Lazarus MD, Marschner I, Barter CE. Exertional oxygen of limited benefit in patients with chronic obstructive pulmonary disease and mild hypoxemia. Am J Respir Crit Care Med 1995; 152: 16161619.

115. Rooyackers JM, Dekhuijzen PN, Van Herwaarden CL, Folgering HT. Training with supplemental oxygen in patients with COPD and hypoxaemia at peak exercise. Eur Respir J 1997; 10: 1278-1284.

116. Wadell K, Henriksson-Larsen K, Lundgren R. Physical training with and without oxygen in patients with chronic obstructive pulmonary disease and exercise-induced hypoxaemia. J Rehabil Med 2001; 33: 200-205.

117. Emtner M, Porszasz J, Burns M, Somfay A, Casaburi R. Benefits of supplemental oxygen in exercise training in nonhypoxemic COPD patients. Am J Respir Crit Care Med 2003; 68: 1034-1042.

118. Serres I, Varray A, Vallet G, Micallef JP, Prefaut C. Improved skeletal muscle performance after individualized exercise training in patients with chronic obstructive pulmonary disease. J Cardiopulm Rehabil 1997; 17: 232238.

119. Carrieri-Kohlman V, Stulbarg MS. Dyspnea: assessment and management. In: Hodgkin JE, Celli BR, Connors GL, eds. Pulmonary rehabilitation. Guidelines to success. Philadelphia, Lippincott, Williams and Wilkins, 2000; pp. 57-89.

120. Esteve F, Blanc-Gras N, Gallego J, Benchetrit G. The effects of breathing pattern training on ventilatory function in patients with COPD. Biofeedback Self Regul 1996; 21: 311321.

121. Tiep BL, Burns M, Kao D, Herrera J, Madison R. Pursed lips breathing training using ear oximetry. Chest 1986; 90: 218-223.

122. Holliday JE, Hyers TM. The reduction of weaning time from mechanical ventilation using tidal volume and relaxation biofeedback. Am Rev Respir Dis 1990; 141: 1214-1220.

123. Collins E, Fehr 1, Bannert C, et al. Effect of ventilationfeedback training on endurance and perceived breathlessness during constant work-rate leg-cycle exercise in patients with COPD. J Rehab Res Devel 2003; 40: 35-44. 